\title{
Extended visual appearance texture features
}

\author{
Simon-Frédéric Désage ${ }^{a}$, Gilles Pitard ${ }^{a}$, Maurice Pillet $^{a}$, Hugues Favrelière $^{a}$, Jean-Luc Maire $^{a}$, $^{-}$ \\ Fabrice Frelin $^{a}$, Serge Samper ${ }^{a, b}$ and Gaëtan Le Goïc ${ }^{a, c}$ \\ ${ }^{a}$ SYMME, Laboratoire des Systèmes et Matériaux pour la Mécatronique, Université de Savoie, \\ Annecy, France; \\ ${ }^{b}$ LARMAUR - ERL CNRS 6274, Laboratoire de Recherche en Mécanique Appliquée de \\ l'Université de Rennes 1, Rennes, France; \\ ${ }^{c}$ LE2I, Laboratoire d'Electronique, Informatique et Image, UMC CNRS 6306, Université de \\ Bourgogne, Auxerre, France
}

\begin{abstract}
The research purpose is to improve surface characterization based on what is perceived by human eye and on the 2006 CIE report. This report defines four headings under which possible measures might be made: color, gloss, translucency and texture. It is therefore important to define parameters able to discriminate surfaces, in accordance with the perception of human eye. Our starting point in assessing a surface is the measurement of its reflectance (acquisition of ABRDF for visual rendering), i.e. evaluate a set of images from different angles of lighting rather than a single image. The research question is how calculate, from this enhanced information, some discriminating parameters. We propose to use an image processing approach of texture that reflects spatial variations of pixel for translating changes in color, material and relief. From a set of images from different angles of light, we compute associated Haralick features for constructing new (extended) features, called Bidimensional Haralick Functions (BHF), and exploit them for discriminating surfaces. We propose another framework in three parts such as color, material and relief.
\end{abstract}

Keywords: Soft metrology, visual inspection, BTF, ABRDF, Texture, Haralick features, BHF, surface characterization

\section{INTRODUCTION}

\subsection{Motivation}

Nowadays, perceived quality of visual appearance is [still] an industrial matter. The main difficulty is to link objective measurements and subjective human aesthetic indicators. The CIE (International Commission on Illumination) provided a technical report CIE 175:2006 "A framework for the measurement of visual appearance". ${ }^{1}$ This report defines four headings under which possible measures might be made: color, gloss, translucency and texture. However, when a customer, and thus imitating his behavior, a human controller, inspect the visual appearance of a product, they can not differentiate the four parties. Considering color and translucency as known and controlled by the industry, we have chosen to work on the gloss and texture to quantify the impact on the human perception. This paper is then an echo of a "soft metrology" approach, ${ }^{2}$ because this paper presents some measurements to compare with the visual human behavior. A known difficulty is the interdependence of these measurements because translucency can influence color, which may influence gloss, and texture is probably a combination of all three. ${ }^{3}$ Our starting point is the study and the imitation of human behavior in the surface quality inspection (Figure 1).

\footnotetext{
Further author information: (Send correspondence to Simon-Frédéric Désage)

E-mail: simon-frederic.desage@univ-savoie.fr

E-mail:\{gilles.pitard, maurice.pillet, hugues.favreliere, jean-luc.maire, fabrice.frelin\}@univ-savoie.fr

E-mail: serge.samper@univ-rennes1.fr and gaetan.le-goic@u-bourgogne.fr
} 


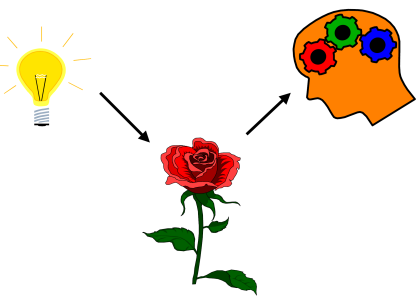

Figure 1: The vision scheme from $\mathrm{CIE}^{1}$

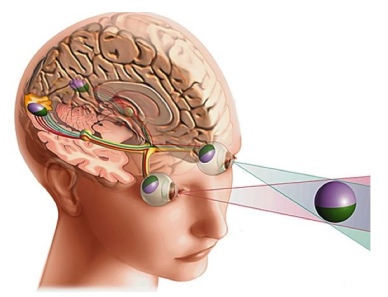

Figure 2: The human visual system for surface inspection. Credit: Michel Saemann for Larousse

\subsection{Methodology}

From surface quality inspection, the reflectance problem is a simple problem between three parts : light, the studied surface and the observer. Indeed, the formalization of human quality inspection consists of three main phases : Exploration, Evaluation and Decision. ${ }^{4}$ There are only two equivalent interactions defining the human perceptual system with the visual surface appearance (Figure 2). The first interaction corresponds to the one between visual surface appearance and the human eye, and the second one between scanned surface and the human brain. We propose to follow these interactions to construct objective visual texture features. For imitating the first interaction, a goniophotometer is used for optic datas acquisition and the exploitation of most advanced visual surface representation allows to the link with human eye. The first part describes the use of digital surface appearance and the measurement instrument. The second part of article recalls classical image processing approach, which allows to human-like texture classification.

The interest of this paper, described in the third part, is the combination of two previous approaches to describe better surfaces and their gonio-appearances in relation to human perception. The problem relates to the characterization of complex surfaces, such as for surfaces having the same hue, the same relief and different material.

\section{DIGITAL SURFACE REPRESENTATION}

Some recent works have shown different representations of visual surface as appearance measurement. Here, we would like to highlight the book "Visual Texture" ${ }^{5}$ of Mr Haindl and Mr Filip is remarkably clear about the reflectance problem, and which lists these recent works. We remind taking direct their definitions of the General Reflectance Function (GRF) (Equation 1 and Figure 3), their reflectance functions Taxonomy (Figure 4) and the model corresponding to our measurement system : the Surface Reflectance Field (SRF) (Figure 5.)

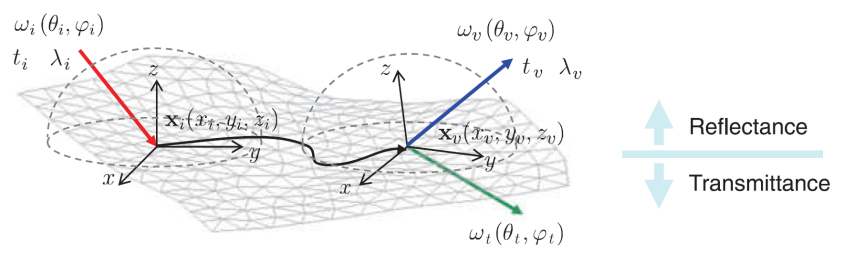

Figure 3: The General Reflectance Model ${ }^{5}$

Physically, the phenomenon is described by a very complex function of 16 variables.

The general reflectance function $(\mathrm{GRF})^{5}$ has 16 dimensions (16D):

$$
Y_{r}^{G R F}=\operatorname{GRF}\left(\lambda_{i}, x_{i}, y_{i}, z_{i}, t_{i}, \theta_{i}, \varphi_{i}, \lambda_{v}, x_{v}, y_{v}, z_{v}, t_{v}, \theta_{v}, \varphi_{v}, \theta_{t}, \varphi_{t}\right)
$$

where $r=\left[r_{1}, \ldots, r_{16}\right]$ is the multi-index with corresponding partial indices. All possible values of the index will be denoted by •, e.g., a color input spectrum in the RGB space $Y_{\bullet, r_{2}, \ldots, r_{16}}=\left[Y_{R, r_{2}, \ldots, r_{16}}, Y_{G, r_{2}, \ldots, r_{16}}, Y_{B, r_{2}, \ldots, r_{16}}\right]$ and the missing index by $\phi$, e.g., a monospectral input $Y_{\phi, r_{2}, \ldots, r_{16}}$. 
GRF describes the incident light with spectral value $\lambda_{i}$; illuminating surface location $x_{i}, y_{i}, z_{i}$ in time $t_{i}$; under spherical incidence angles $\omega_{i}=\left[\theta_{i}, \varphi_{i}\right]$ and observed at time $t_{v}$ from surface location $x_{v}, y_{v}, z_{v}$ under spherical reflectance angles $\omega_{v}=\left[\theta_{v}, \varphi_{v}\right]$ and spectrum $\lambda_{v}$; here $\omega_{t}=\left[\theta_{t}, \varphi_{t}\right]$ are the corresponding transmittance angles where $\omega=[\theta, \varphi]$ are the elevation and azimuthal angles, respectively. The model height parameters $z_{i}, z_{v}$ indicate that radiance along light rays is not constant but depends on the height. The GRF function (Equation 1) is too complex to be accurately measured or modeled, hence some simplifying assumptions are inevitable in any practical application. The taxonomy of simplifying assumptions can be divided into two subgroups based on the possibility of neglecting a surface texture.

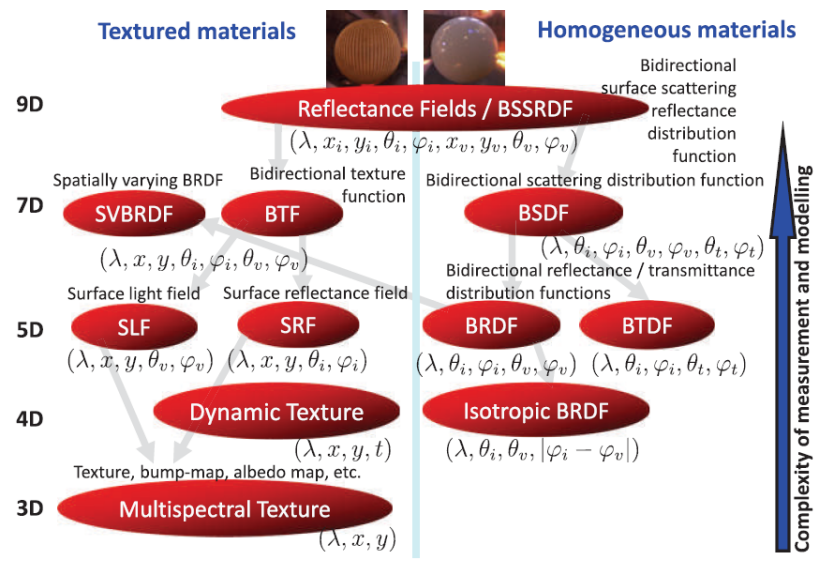

Figure 4: The General Reflectance Taxonomy ${ }^{5}$

We also share the definition of a visual texture, which is a resolution-based relative notion. The scale of observation is important because any natural surface material is textured and our perception of surfaces as textured or smoothly homogeneous (i.e., non-textured) only depends on the corresponding surface resolution. The same surface observed from a distance can be categorized as smooth, while its close observation may reveal rough-textured surface. The GRF simplifying taxonomy (Figure 4) is obviously not exhaustive, but it allows the reader to quickly locate themselves in relation to the range of possibilities, and locate the model used. Two particular features may correspond to an industrial system, with limited degrees of freedom because to meet industrial requirements, it is necessary to fix extent possible the maximum settings.

In our vision problem, we have three sources of settings: the light source, the study area and the viewpoint. In the general cases of inspection systems, the study position of the object is fixed and at least the position of another between the light source and the viewpoint. If the light source position is fixed, a Surface Light Field (SLF) model-like function is measured and verify the equation 3, as the figure 6 shows. On the contrary, if the viewpoint is fixed, a Surface Reflectance Field (SRF) model-like function is measured and verify the equation 2 , as the figure 5 shows. Between to multiply the light sources or viewpoints, the calibration protocol such as financial stress have occurred in our choice. In other words, a light source costs less than a photonic sensor and is easier to calibrate, so we opted for a system complying with the surface light field model.

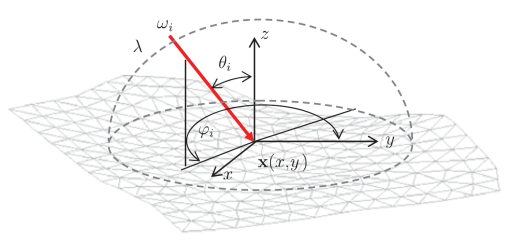

Figure 5: Surface Reflectance Field Model ${ }^{5}$

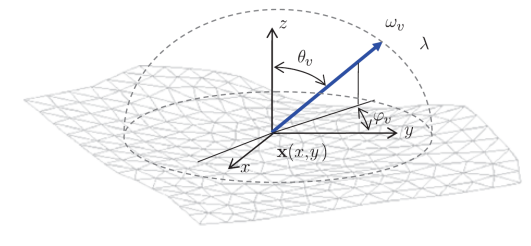

Figure 6: Surface Light Field Model ${ }^{5}$ 
The Surface Reflectance Field $(\mathrm{SRF})^{5}$ has then as 5 dimensions (5D) with a fixed viewpoint and a free light position :

$$
Y_{r}^{S R F}=G R F\left(\lambda, x, y, \theta_{i}, \varphi_{i}\right)
$$

The Surface Light Field $(\mathrm{SLF})^{5}$ is a similar function of SRF, with a fixed incident light and a free observer :

$$
Y_{r}^{S L F}=\operatorname{GRF}\left(\lambda, x, y, \theta_{v}, \varphi_{v}\right)
$$

To be rigorous physical part of the system used, we took into account assumptions. We only mention the following assumptions, useful to simplify the SRF model:

A1 light transport takes zero time $\left(t_{i}=t_{v}\right.$ and $\left.t_{v}=\phi\right)$

A2 reflectance behavior of the surface is time invariant $\left(t_{i}=t_{v}=\right.$ const., $\left.t_{i}=t_{v}=\phi\right)$

A3 interaction does not change wavelength $\left(\lambda_{i}=\lambda_{v}\right.$, i.e., $\left.\lambda_{v}=\phi\right)$

A 4 constant radiance along light rays $\left(z_{i}=z_{v}=\phi\right)$

A5 no transmittance $\left(\theta_{v}=\varphi_{t}=\phi\right)$ (no simultaneous reflectance and transmittance)

A6 incident light leaves at the same point $x_{i}=x_{v}, y_{i}=y_{v}\left(x_{v}=y_{v}=\phi\right)$

A13 fixed illumination $\left(\theta_{i}=\right.$ const., $\varphi_{i}=$ const. $)$; For SLF only

A14 fixed viewing angle $\left(\theta_{v}=\right.$ const., $\varphi_{v}=$ const.); For SRF only

\subsection{Acquisition}

In industrial terms of quality control, the Commission Internationale de l'Eclairage (CIE) recommends lighting conditions such as the type of light and its position on the surface (Figure 7). The standard illuminant is D65 ${ }^{1}$ (Figure 8). There are other experimental measurement protocols to identify the specularity type ${ }^{6}$ of the color of specular surfaces. ${ }^{7}$

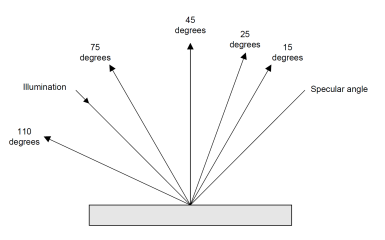

Figure 7: The acquisition system ${ }^{1}$

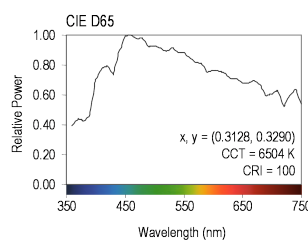

Figure 8: The D65 illuminant spectrum ${ }^{1}$

In practice, for aesthetic quality inspection, the light having its defined position, the human controller freedoms are the position of the observed surface and the relative position of his eye. It usually turns out that this is the observed surface that changes the position more because the controller has the object in his hand and played with lighting to highlight the differences of aspects to his eye. Three effects ${ }^{4}$ are wanted for surface inspection: the brilliant effect (Figure 9 (a)), the black light effect (Figure 9 (b)) and the mirror effect (Figure 9 (c)).

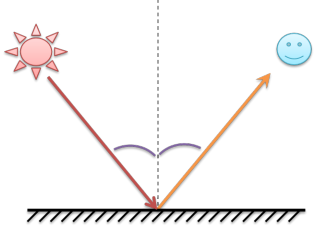

(a)

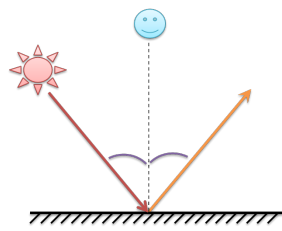

(b)

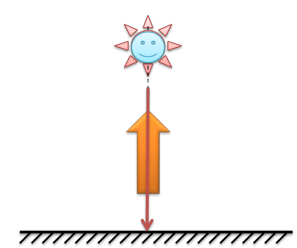

(c)

Figure 9: (a) The brilliant effect (b) The black light effect (c) The mirror effect 
We propose to use a similar method for gloss measurement with a recent visual texture representation called Bidimensional Texture Function (BTF) ${ }^{8}$ (Figure 10), because it is the closest function of controller observation. One difficulty for controller is to repeat the same light path and the same observation function, because he can not realise the whole BTF. In pratice, each human inspection is a part of BTF. Our bias is to achieve a similar and regular inspection for each surface. We set the position of viewpoint and the relative position of light sources to be used to perform the structured lighting sequence (Figure 11) for the chosen SRF model (Figure 5).

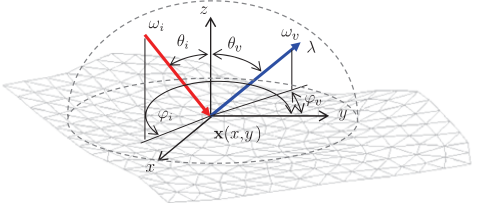

Figure 10: The BTF model ${ }^{5}$

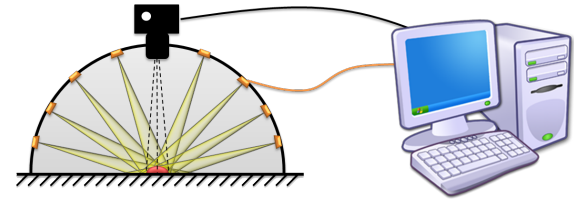

Figure 11: The acquisition system

Wanting to characterize some textured surface, the measured function for one pixel is called Apparent Bidimensional Reflectance Distribution Function (ABRDF). This function is called apparent because it can violate either of two basic BRDF properties, i.e., view and illumination direction reciprocity or energy conservation. This behavior is caused by the surface relief, such as self-shadowing, self-occlusions, subsurface scattering and other complex effects occurring in the material structure which are not represented in the true BRDF models.

\subsection{Reconstruction}

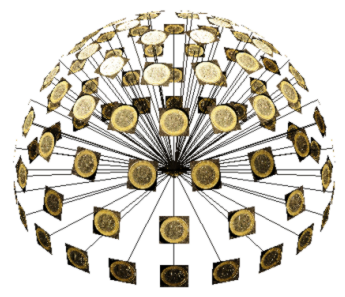

(a)

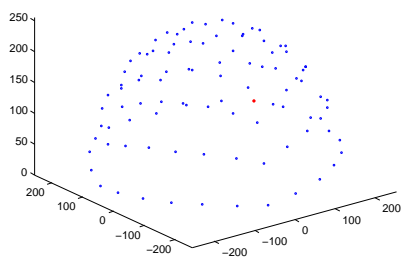

(b)

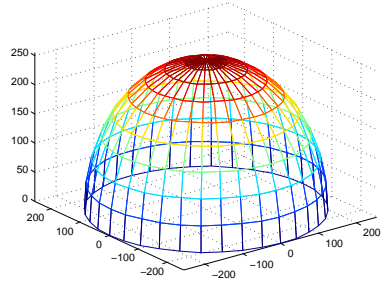

(c)

Figure 12: (a) The acquired images sequence (b) The measured points cloud for one pixel (c) The ABRDF function

From the images sequence (Figure $12(\mathrm{a})$ ), we can obtain useful cloud points by reprojection of pixel values on the axis of corresponding incident light (Figure 12 (b)). For editing the useful ABRDF, we use a pixel-wise modeling method called Polynomial Texture Maps (PTM) ${ }^{9}$ There are other methods ${ }^{5}$ identified and more efficient such as Hemispherical Harmonic (HSH) ${ }^{10}$ but we start with the simpler method. We mention these methods as principle for the reconstruction method of hemishperic function and we can not discuss of reconstruction performances. The reconstruction illustration corresponds to the ideal example of a Lambertian surface (Figure 12 (c)) irrespective of the light incident function on the surface.

The PTM method models illumination dependence of individual pixels using the following pixel-wise biquadratic formula :

$$
S R F_{v}(r, i) \approx a_{0}(r) u_{x}^{2}+a_{1}(r) u_{y}^{2}+a_{2}(r) u_{x} u_{y}+a_{3}(r) u_{x}+a_{4}(r) u_{y}+a_{5}(r)
$$

where $u_{x}, u_{y}$ are projections of the normalised light vector into the local coordinate system $r=(x, y)$. The set of $n_{i}$ pixels is considered as reflectance data, where $i=1, \ldots, n_{i}$ is the illumination position index and $v$ is the actual view position index $v=1, \ldots, n_{v}$. The $n_{p}=6$ polynomial coefficients $a_{0}-a_{5}$ are fitted in each pixel by means of singular value decomposition. From these coefficients, the approximated function is known. 


\subsection{Extraction}

Once reconstructed, each ABRDF can be used for calculate the surface appearance corresponding for any incident light position from superior hemispheric space. Whereas ABRDF has not the physic sense like a "true" BRDF, the shape of ABRDF is may be seen as the Phong model (Figure 13 (a) and (c)).

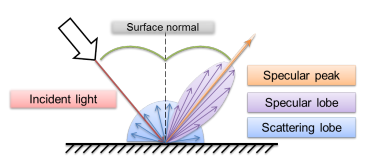

(a)

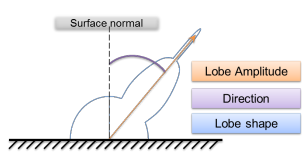

(b)

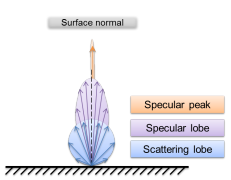

(c)

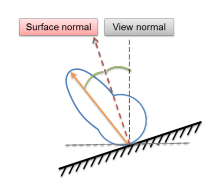

(d)

Figure 13: (a) The Phong BRDF model (b) Phong-based Surface appearance framework (c) Adapted Phong model to ABRDF (d) ABRDF-based Surface appearance framework

We propose a surface appearance framework from ABRDF slice in three parts: color, material and relief. The shape of ABRDF slice is a feature of material, respectively the power/amplitude for the color (Dependent of each wavelength), and the direction for the relief related to the optical law of Snell-Descartes (Figure 13 (a)). The amplitude corresponds to the maximum of function and the function direction is the direction of maximum such as the specular direction. We can extract three types of image for each information type from ABRDF slice. This framework (Figure 14) can help to discuss about visual texture definition. A photography, as image texture, is a visual texture slice and it is a local result of color, material and relief combination. There is surface texture such as only relief texture.

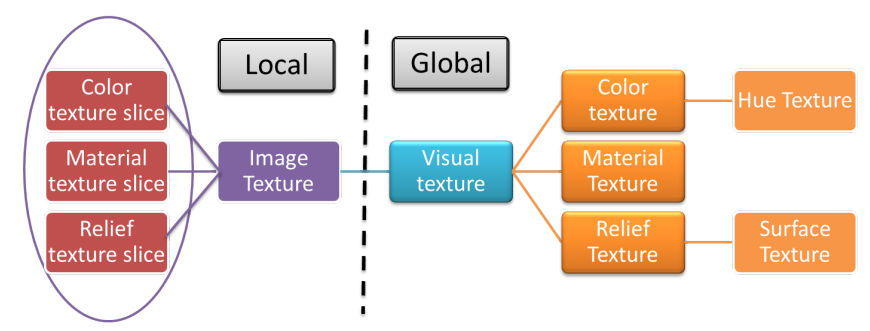

Figure 14: Texture framework

One image combines these three types of information whereas we can distinguish three sub-textures from ABRDF slice (Figure 13). For quality inspection, the advantage is to guarantee to find directly visual anomaly and their causes. The right image is the one with the anomalies if they exist. Then, we must characterize each image - photography or numerical representation for each visual texture component.

\section{TEXTURE CLASSIFICATION}

Some works have shown the useful of Haralick features for texture classification. ${ }^{11}$ The difficulty to get a good classification was to have the "good" image of the product surface, i.e. an image with a uniform or well directed lighting and a uniform or well directed viewing. For quality inspection, the right image is the one with the anomalies if they exist, even if lighting is not uniform. Of course, it is necessary to have a suitable resolution. So, it is necessary to identify the image contents. Each method is used for one image. 


\subsection{Co-occurrence matrix}

Several works are identified as using gray-level co-occurrence matrix (GLCM) method for defect detection. ${ }^{12}$ The co-occurrence matrix method is a statistical method for textural defect detection. There are other statistical methods such as histogram properties, local binary pattern (LPB) or autocorrelation. There are also other methods: structural methods, filter-based or model based methods ${ }^{12} .^{13}$ In this paper, we focus on the cooccurrence matrix method to ease the demonstration.

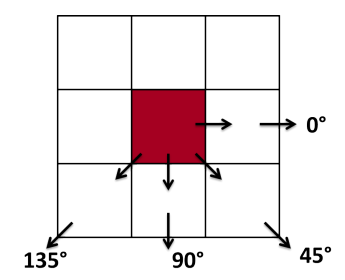

Figure 15: The 4 directions considered with a step of 1 pixel

As classical processing, Haralick features used to describe a single image. We do a recall of the generic method to compute a GLCM and applied Haralick features, ${ }^{11}$ while GLCM method is one of the most well-known and widely used texture features. GLCM measures the spatial dependency of two gray-levels, given a displacement vector. The classical displacement has a size of 1 pixel, but it can be adapted in function of texture frequency. There is 4 directions in an image (with a square matrix unit). There are many interpretations of co-occurrence matrix. The most common method is to use sum of 4 directions matrix.

$$
m[I, \theta](i, j)=\operatorname{Card}\left\{\{p, p+t\} \epsilon I^{2} ; f(p)=i, f(p+t)=j \text { and } \operatorname{dir}(t)=\theta\right\}
$$

where $I$ denotes the image, $f$ is its associated function, $\theta$ the transition direction, $i, j$ are gray-levels, $p$ is the start pixel and $t$ denotes transition step to get the second pixel.

To be rotation invariant in the square matrix of pixels, we propose to use the sum of the 4 directions matrix such as :

$$
M[I]=\sum_{n t=1}^{4}\left(m[I, \theta]_{\theta=45 *(n t-1)}(i, j)_{i \epsilon\left[0 . .2^{N}\right], j \epsilon\left[0 . .2^{N}\right]}\right)
$$

where $N$ is the gray-levels number.

Some examples of co-occurrence matrix calculations are shown in the following figures 16 (a), (b), (c) and (d). The first example shows for a reduced image with a 5 by 5 pixels size and 5 graylevels, the calculation method in the $0^{\circ}$ direction. The 4 to 2 and 3 to 4 transitions are highlighted such as there are 2 transitions 4 to 2 and 3 transitions 3 to 4 . The second example is the same principle with the same reduced image, except that we consider transitions in 4 directions.

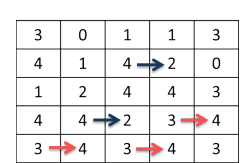

(a)

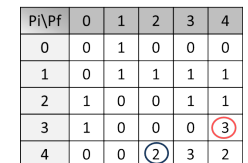

(b)

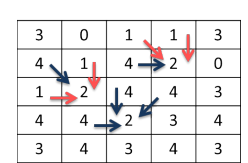

(c)

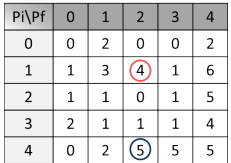

(d)

Figure 16: (a) Some transitions for $0^{\circ}$ direction (b) GLCM for $0^{\circ}$ direction (c) Some transitions for 4 directions (d) GLCM sum for 4 directions 


\subsection{Haralick features}

It does not need to mention all Haralick features. ${ }^{11}$ There are also other features ${ }^{14}$ set out more recently to describe a co-occurrence matrix. For principle, our demonstration will be limited to typical and the most used in the litterature attributes, such as energy, contrast and homogeneity. One must know that some attributes have been the subject of intuitive analysis in their contribution to characterization of textures. ${ }^{14}$

- Feature number 01 : Angular Second Moment or Energy

$$
f_{01}=\sum_{i=0}^{N-1} \sum_{j=0}^{N-1}\{M[I, t](i, j)\}^{2}
$$

- Feature number 02 : Contrast

$$
f_{02}=\sum_{n=0}^{N-1} n^{2}\left\{\sum_{i=0}^{N-1} \sum_{j=0}^{N-1} M[I, t](i, j)\right\}
$$

- Feature number 05 : Inverse Difference Moment or Homogeneity

$$
f_{05}=\sum_{i=0}^{N-1} \sum_{j=0}^{N-1} \frac{1}{1+(i-j)^{2}} M[I, t](i, j)
$$

The approach is to process an image of a surface (Figure 17 (a) and (d)), so as to get his co-occurrence matrix (Figure 17 (b) and (e)) that highlights a particular distribution of pixel to pixel transitions. To characterize this distribution, we use the calculation of the characteristics (Figure 17 (c) and (f)). In our case, the obtained values have no special meaning, they only serve to illustrate the approach.

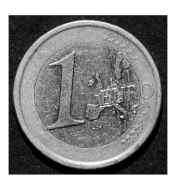

(a)

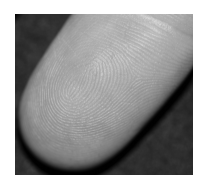

(d)

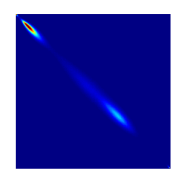

(b)

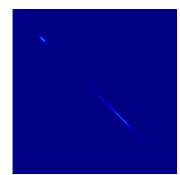

(e)

\begin{tabular}{|c|c|}
\hline Feature & Value \\
\hline Energy & $0.335 \mathrm{E}-03$ \\
\hline Contrast & 189 \\
\hline Homogeneity & $157 \mathrm{E}-03$ \\
\hline
\end{tabular}

(c)

\begin{tabular}{|c|c|}
\hline Feature & Value \\
\hline Energy & $2.32 \mathrm{E}-03$ \\
\hline Contrast & 2.67 \\
\hline Homogeneity & $558 \mathrm{E}-03$ \\
\hline
\end{tabular}

(f)

Figure 17: (a) (resp.(d)) One example image (b) (resp.(e)) The associated GLCM (c) (resp.(f)) The associated features value

\section{PROPOSED APPROACH}

A human controller scans different lighting and viewing positions to get the right image. The right image is the one with the anomalies if they exist. Hence, the idea is to stick to the controller behavior and collect all the useful images of surface. However, it is necessary to process this new and enriched data relative to a single image. We present the (extension) adaptation of Haralick to BTF and some results of Bidimensional Haralick Functions (BHF). 
The ideal case will be to compute co-occurrence matrix from exact ABRDFs (or maybe their coefficients if they are exacts too) and their Haralick features, but it is [too (for the moment)] complex. So the idea is to do the inverse, i.e., to compute co-occurrence matrix for acquired images sequence and their Haralick features, for reconstructing a bidimensional function of Haralick features.

The data volume does not allow to present each result. We have chosen to give the preview of theoretical cases to present the generic method.

The demonstration will be focus on simple and interesting cases. Before to process complex cases where surface has many different subtextures, we process with uniform textures. The interesting cases are ones which have goniovariance, i.e. an anisotropic appearance.

\subsection{Received incident light function}

Some cases, such as a lambertian and plane surface, require no new feature since they are isotropic, and this is true if the lighting incident is isotropic, i.e., compensated for the surface to receive the same amount of light regardless of the impact. Otherwise, for these, it will be sufficient to calculate Haralick features once to know the magnitude of the associated hemispherical BHF.

Considering the previous A4 assumption about constant radiance, there are two possible interpretations. The first interpretation is each lighting source emits the same quantity of light (Figures 18 (a), (b) and (c)), and the second interpretation is the surface receives as much light for each source (Figures 18 (d)). Only the second interpretation is isotropic. Each interpretation defines one received lighting function, which influences the measured appearance behavior of surface.

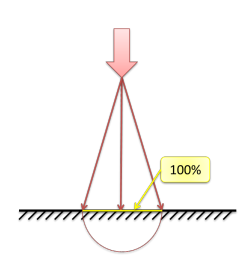

(a)

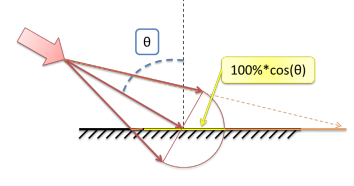

(b)

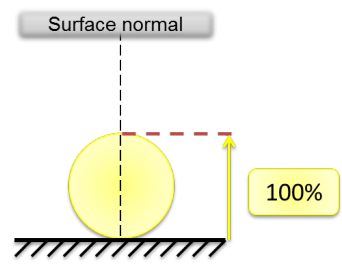

(c)

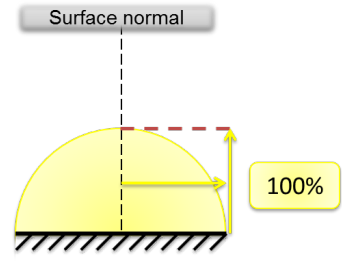

(d)

Figure 18: (a) Illumination received by a unit area for a light source disposed to the surface normal (b) Respectively in (a) to a source having a direction inclined with respect to the normal (c) Slice of received illumination function for constant sources (d) Slice of compensated illumination function for a constant radiance

The first interpretation was considered because it is the easiest system with all sources emit the same light intensity. There is then a second parameter, depending on the surface, which influences the received light function. This is the relief of the surface. Indeed, it is observable from the figure 18 (c) that the received illumination sphere is oriented normal to the surface. Then, two (simple) reliefs are taken for example : a plane surface (Figure 19 (a)) and a no plane surface (Figure 19 (c)). There is shown the relief effect on the received lighting function, in the following figures 19 (b) and (d). The measured intensities are done in a superior hemisphere for horizontal surface. If a surface is oriented, it lacks a function piece, which is outside the superior hemisphere.

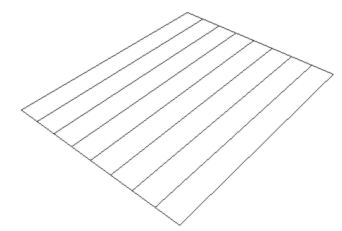

(a)

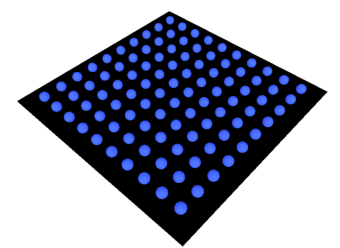

(b)

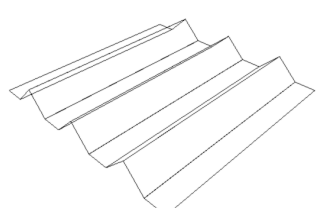

(c)

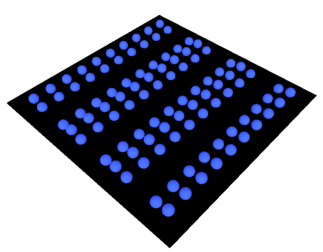

(d)

Figure 19: (a) Plane relief (b) Surface plane and Lambertian (c) No plane relief (d) Lambertian surface with no plane relief 


\subsection{Study cases}

Considering the previous framework : Material, color and relief, the demonstration show different cases with some states for each parameter. As shown previous (Figure 19), we consider two relief. It is normal to consider equally two color levels and two material levels. For color levels, there are a case with the same graylevel and a case with a graylevels gradient, as differences between Figures 20 (a) and (b). For material, the difference is made between the behavior : the first case is Lambertian, i.e., totally diffusing, and the second is only specular as a metal, such as Figure 13 show generic BRDF decomposition.

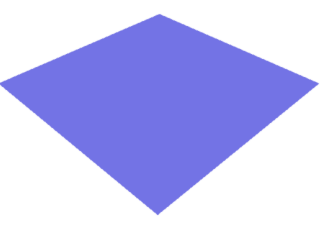

(a)

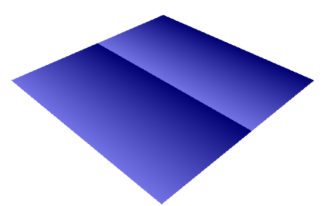

(b)

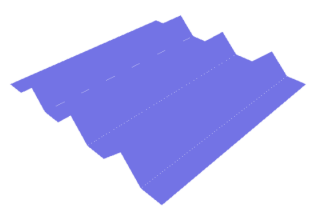

(c)

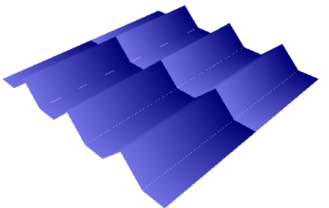

(d)

Figure 20: (a) Plane and uni-color (b) Plane and color gradient (c) No plane relief and uni-color (d) No plane and color gradient

All the considered cases are illustrated in figure 21. For ease of reading, we list the various cases such as the figure 21 shown rather than listing each associate parameter. This figure can serve as a basis for a design of experiment in connection with the BHF values (Figure 28). The studied cases are relatively restrictive to facilitate the demonstration, but it would be interesting to test this new characterization for all combinations of parameters Material, Relief and Color. Selected cases have a repeated pattern start to contain a beginning of texture. It is interesting because the unitary surface, sometimes called "texton", reacts similarly to a larger area composed of multiple unit areas.

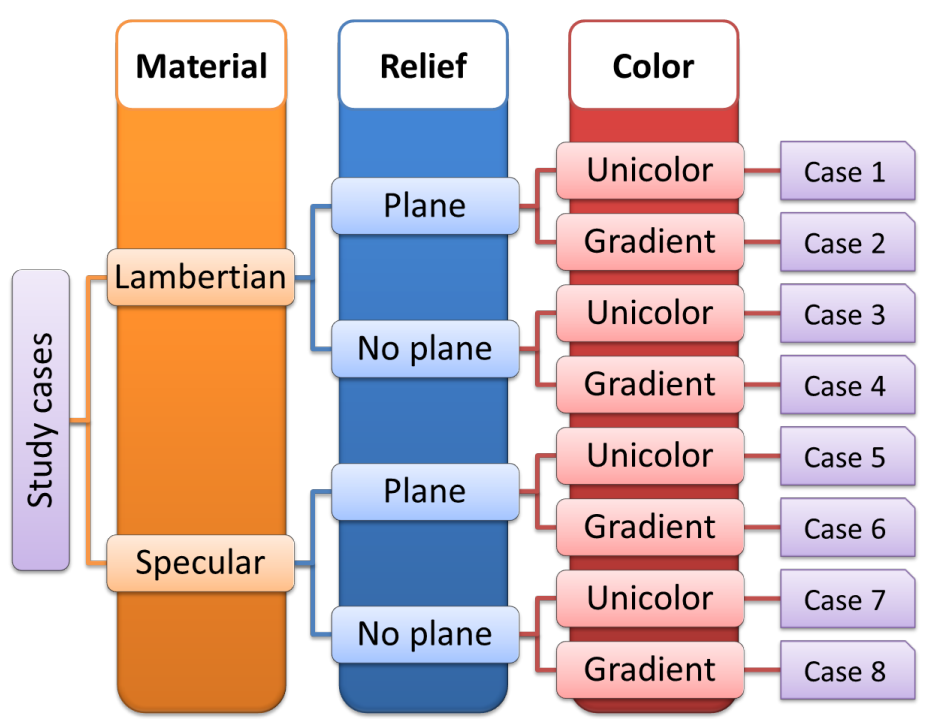

Figure 21: The study cases scheme 


\subsection{Lambertian case}

We deal with cases of Lambertian surfaces, called cases 1 - 4 (Figure 21), with their BRDF which is the natural reflectance behavior of the surface (Figure 22), and their ABRDF (Figure 23) which is the combination of BRDF and lighting function.

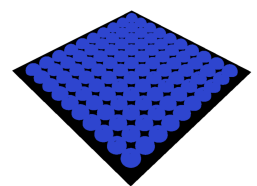

(a) Case 1

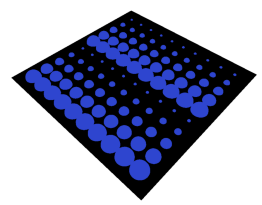

(b) Case 2

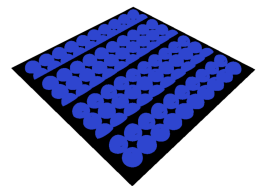

(c) Case 3

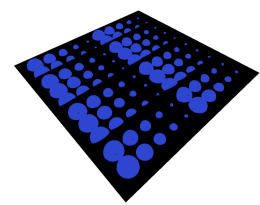

(d) Case 4

Figure 22: BRDF field for Lambertian surface

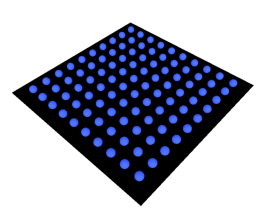

(a) Case 1

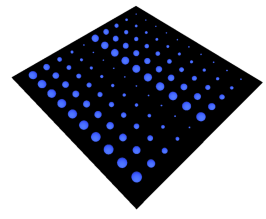

(b) Case 2

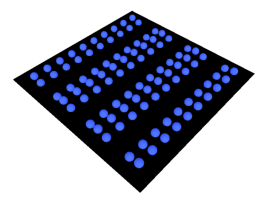

(c) Case 3

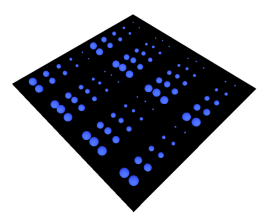

(d) Case 4

Figure 23: ABRDF field for Lambertian surface

\subsection{Specular case}

We deal with cases of specular surfaces, called cases 5 - 8 (Figure 21), with their BRDF which is (still) the natural reflectance behavior of the surface (Figure 24), and their ABRDF (Figure 25) which is the combination of BRDF and lighting function. The BRDF and ABRDF fields are similar to Lambertian cases except that the basic function is a lob rather than a sphere. This induces a change of more sudden constrate and therefore a stronger appearance disparity for no plane surfaces. Some works have already seen the importance of lob shape and size in the glossy appearance measures for recognizing real materials. ${ }^{6}$

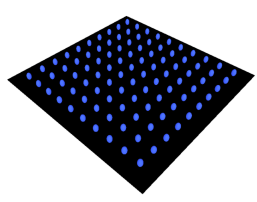

(a) Case 5

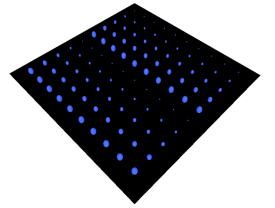

(b) Case 6

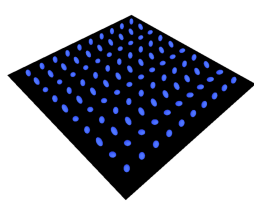

(c) Case 7

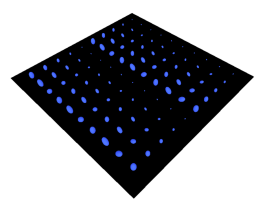

(d) Case 8

Figure 24: BRDF field for specular surface

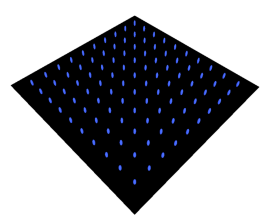

(a) Case 5

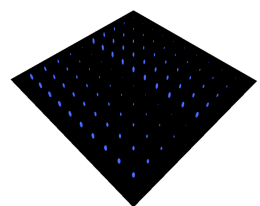

(b) Case 6

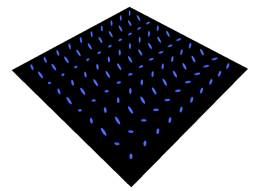

(c) Case 7

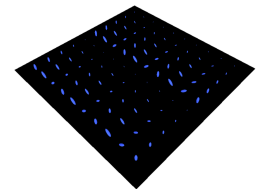

(d) Case 8

Figure 25: ABRDF field for specular surface 


\subsection{Bidimensional Haralick Functions}

We take homogeneity for example, because it is a known parameter as relevant for quality assessment, particularly in the perceptual quality of printed surfaces. ${ }^{15}$ It is interesting to observe that the transformation of the Lambertian or specular surfaces BHF are not the same as the surfaces are subject to the same changes (Figure 26). These features of transformation can then be considered as a signature. Otherwise, the results of the cases 4 and 8 , in the figure 26, are likely the result of the combination, respectively, cases 2 and 3 , and cases 6 and 7 .

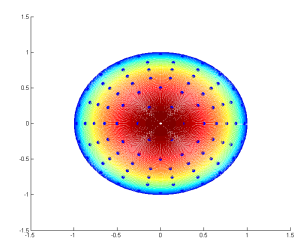

(a) Case 1

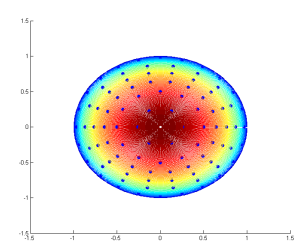

(e) Case 5

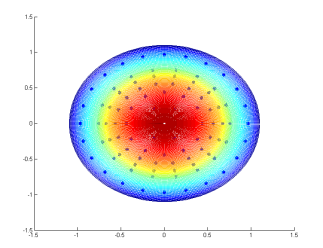

(b) Case 2

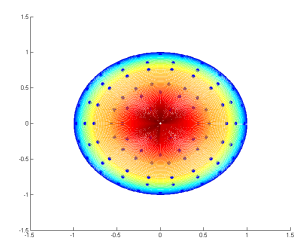

(f) Case 6

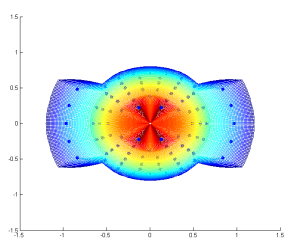

(c) Case 3

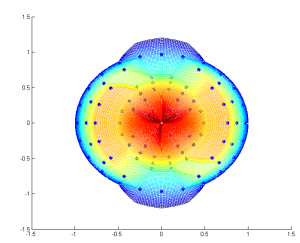

(g) Case 7

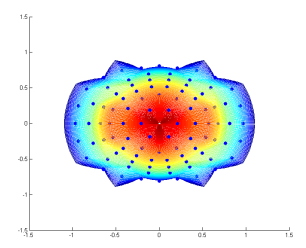

(d) Case 4

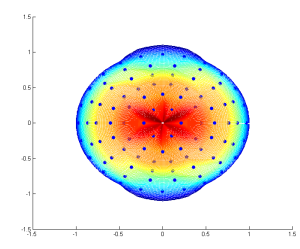

(h) Case 8

Figure 26: Projection of BHF for Homogeneity in the X-Y reference

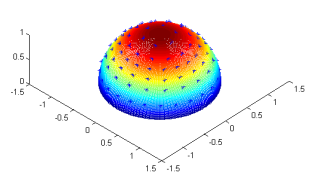

(a) Case 1

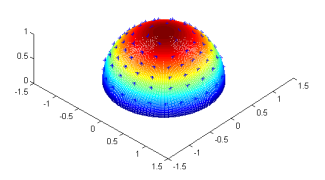

(e) Case 5

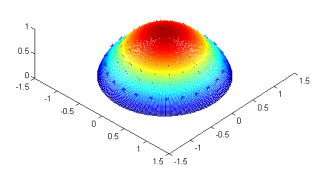

(b) Case 2

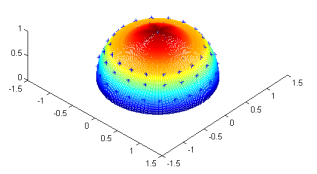

(f) Case 6

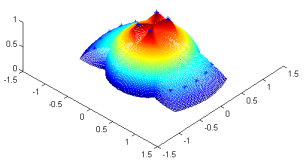

(c) Case 3

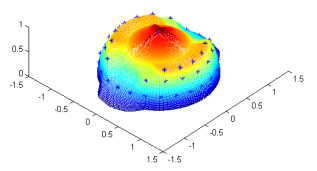

(g) Case 7

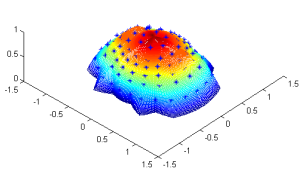

(d) Case 4

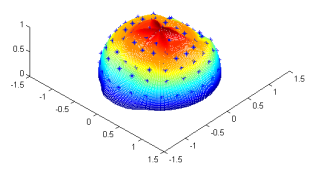

(h) Case 8

Figure 27: Projection of BHF for Homogeneity in the 3D 
The functions in the figures 26 and 27 have their interpolated surfaces to improve visual distinctness of BHF transformations. If we need compute a distance between BHF, we propose to use the associated coefficients from an approximation, such as polynomial approximation (Equation 4) gives respective decompositions shown in figure 28. The cases 1 and 5 are identical according to homogeneity, suddenly their coefficients are superimposed. Clearly, the fourth polynomial coefficient seems pointless to classify different cases.

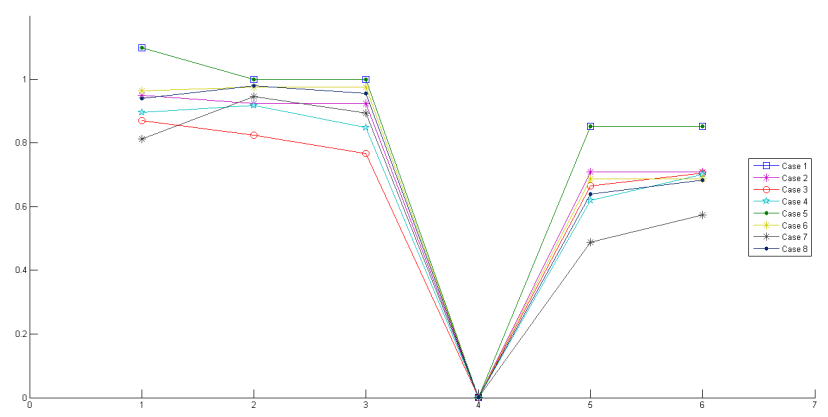

Figure 28: Polynomial decomposition coefficients corresponding to respective BHF

\section{CONCLUSION AND PERSPECTIVES}

We have proposed a combined method for surface characterization through an image processing approach and scanning their complete visual texture, i.e., for the surface appearance seen under an illumination hemisphere.

The method provides enriched features compared to the conventional method. Visual trend of these new features is that they can help distinguish visual texture into three sub-textures related relief, material and color. In this sense, this trend should be confirmed with the application of the method on a larger variety of surfaces and taking into account a significant number of features (at least all Haralick features, for example). The application could be a design of experiments with real samples.

There are three perspectives of this work for visual inspection. The first perspective is when visual inspection is applied to aesthetic field, there are two challenges:

1 - Have a Repeatable and Reproducible (R\&R) method. ${ }^{16}$

2 - Have a human perception-like processing with visual attributes.

We can use some elements of this paper for establishing features of visual data. This data can be used for trying to understand the human visual behavior, because we can verify uniformity and reproducibility of visual exploration and evaluation data. The second perspective is for automatic defect classification. In fact, some works have shown the useful of Haralick features for automatic defect classification from different co-occurrence matrix of one image ${ }^{14} \cdot{ }^{17}$ The third perspective is to use the different well-known methods for editing BTF-like functions, such as Polynomial extended Lafortune Reflectance Model (PLM RF), Reflectance field factorization (PCA RF) or Hemispheric harmonics $(\mathrm{HSH})^{5} \cdot{ }^{10}$

\section{ACKNOWLEDGMENTS}

We thank our SYMME partners in MESURA project as well as Savoie Mont Blanc Industries and Conseil General 74 to enable us to carry out this research by giving us resources. 


\section{REFERENCES}

[1] Pointer, M., "A framework for the measurement of visual appearance," CIE Publication 175-2006: CIE TC1-65 Technical Report (2006).

[2] Krynicki, J.-C., "Introduction to soft metrology," in [XVIII IMEKO World Congress], (2006).

[3] Eugène, C., "Measurement of "total visual appearance" a cie challenge of soft metrology," in [12th IMEKO TC1 83 TC7 Joint Symposium on Man, Science 83 Measurement], 61-65 (2008).

[4] Baudet, N., Maire, J.-L., and Pillet, M., "The visual inspection of product surfaces," Food Quality and Preference 27(2), 153-160 (2013).

[5] Haindl, M. and Filip, J., [Visual Texture: Accurate Material Appearance Measurement, Representation and Modeling], Springer Science \& Business Media (Jan. 2013).

[6] Ged, G., Obein, G., Silvestri, Z., Le Rohellec, J., and Viénot, F., "Recognizing real materials from their glossy appearance," Journal of vision 10(9), 18 (2010).

[7] Charrière, R., Lacaille, G., Pedeferri, M. P., Faucheu, J., and Delafosse, D., "Characterization of the gonioapparent character of colored anodized titanium surfaces," Color Research 83 Application (2014).

[8] Filip, J. and Haindl, M., "Bidirectional texture function modeling: A state of the art survey," IEEE Transactions on Pattern Analysis and Machine Intelligence 31, 1921-1940 (Nov. 2009).

[9] Malzbender, T., Gelb, D., and Wolters, H., "Polynomial texture maps," in [Proceedings of the 28th annual conference on Computer graphics and interactive techniques], 519-528, ACM (2001).

[10] Wang, O., Gunawardane, P., Scher, S., and Davis, J., "Material classification using BRDF slices," in [Computer Vision and Pattern Recognition, 2009. CVPR 2009. IEEE Conference on], 2805-2811, IEEE (2009).

[11] Haralick, R. M., Shanmugam, K., and Dinstein, I. H., "Textural features for image classification," Systems, Man and Cybernetics, IEEE Transactions on (6), 610-621 (1973).

[12] Xie, X., "A review of recent advances in surface defect detection using texture analysis techniques," Electronic Letters on Computer Vision and Image Analysis 7(3), 1-22 (2008).

[13] Nixon, M., [Feature Extraction 83 Image Processing], Academic Press (Jan. 2008).

[14] Porebski, A., Sélection d'attributs de texture couleur pour la classification d'images. Application à l'identification de défauts sur les décors verriers imprimés par sérigraphie., $\mathrm{PhD}$ thesis, Université Lille 1 (2009).

[15] Nébouy, D., Hébert, M., Fournel, T., and Lesur, J.-L., "Visual quality of printed surfaces: Study of homogeneity," in [ISET/SPIE Electronic Imaging], 90160C-90160C, International Society for Optics and Photonics (2014).

[16] Maire, J., Pillet, M., and Baudet, N., "Gage r2\&e2: an effective tool to improve the visual control of products," International Journal of Quality \& Reliability Management 30, 161-176 (Jan. 2013).

[17] Porebski, A., Vandenbroucke, N., and Macaire, L., "Iterative feature selection for color texture classification," in [Image Processing, 2007. ICIP 200\%. IEEE International Conference on], 3, III-509, IEEE (2007). 